\title{
A NOTE ON CERTAIN CLASSES OF CLOSE-TO-CONVEX FUNCTIONS
}

\author{
H. A. AL-KHARSANI
}

\begin{abstract}
The object of the present paper is to derive distortion inequalities for fractional integral operator of functions in the class $K(n, \alpha, \beta)$ consisting of analytic and univalent functions with negative coefficients.
\end{abstract}

\section{Introduction}

Let $A(n)$ denote the class of functions of the form

$$
f(z)=z-\sum_{k=n+1}^{\infty} a_{k} z^{k} \quad\left(a_{k} \geq 0, n \in N=\{1,2,3, \cdots,\}\right)
$$

that are analytic in the unit disk $U=\{z,|z|<1\}$. A function $f(z) \in A(n)$ is said to be in the class $K(n, \alpha, \beta)$ if and only if there exists a function $\varphi(z) \in A(n)$ such that

$$
\left|\frac{\frac{z f^{\prime}(z)}{\varphi(z)}-1}{\frac{z f^{\prime}(z)}{\varphi(z)}+(1-2 \alpha)}\right|<\beta
$$

for some $\alpha(0 \leq \alpha<1), \beta(0<\beta \leq 1)$ and for all $z \in U$. We note that $K(1, \alpha, \beta) \equiv$ $K(\alpha, \beta)$, the class $K(\alpha, \beta)$ was studied by Gupta [1].

A function $f(z) \in A(n)$ is said to be in the class $C^{*}(n, \alpha, \beta)$ if and only if there exists a convex function $\varphi \in A(n)$ such that

$$
\left|\frac{\frac{\left(z f^{\prime}(z)\right)^{\prime}}{\varphi^{\prime}(z)}-1}{\frac{\left(z f^{\prime}(z)\right)^{\prime}}{\varphi^{\prime}(z)}+(1-2 \alpha)}\right|<\beta
$$

for some $\alpha(0 \leq \alpha<1), \beta(0<\beta \leq 1)$ and for all $z \in N$.

It follows immediately that $f(z) \in C^{*}(n, \alpha, \beta)$ if and only if $z f^{\prime} \in K(n, \alpha, \beta)$.

The following coefficient theorem for the class $K(\alpha, \beta)$ will be required in our investigation.

Received May 28, 1998; revised December 28, 1998.

1991 Mathematics Subject Classification. 30C45.

Key words and phrases. Univalent, integral operator, Fractional, convolution. 
Lemma 1. [1]. If $f \in K(\alpha, \beta)$ then

$$
\sum_{k=2}^{\infty}(1+\beta) k a_{k}-(1-\beta+2 \alpha \beta) b_{k} \leq 2 \beta(1-\alpha)
$$

\section{Fractional Integral Operator}

We need the following definition of fractional integral operator given by Srivastave, Saigo and Owa [5]

Definition. Let

$$
F(a, b ; c ; z)=\sum_{k=0}^{\infty} \frac{(a)_{k}(b)_{k}}{(c)_{k}(1)_{k}} z^{k}
$$

where

$$
(\nu)_{k}=\frac{\Gamma(\nu+k)}{\Gamma(\nu)} \begin{cases}1, & k=0 \\ \nu(\nu+1) \cdots(\nu+k-1), & k \in N=\{1,2,3, \cdots\}\end{cases}
$$

For real number $\rho>0, \delta, \eta$, and $\epsilon>\max \{0, \delta-\eta\}-1$, the fractional integral operator $I_{0, z}^{\rho, \delta, \eta}$ is defined by

$$
I_{0, \approx}^{\rho, \delta, \eta} f(z)=\frac{z^{-\rho-\delta}}{\Gamma(\rho)} \int_{0}^{z}(z-t)^{\rho-1}\left(\rho,-\eta ; \rho ; 1-\frac{t}{z}\right) f(t) d t .
$$

where $f(z)$ is a function analytic in a simply connected region of the $z$-plane containing the origin, satisfying

$$
f(z)=O(|z|)^{\epsilon}, \quad z \rightarrow 0,
$$

and the multiplicity of $(z-t)^{\rho-1}=\exp ((\rho-1) \log (z-t))$ where $\log (z-t)$ is supposed to be real when $z-t>0$.

Remark. For $\delta=-\rho$, we note that

$$
I_{0, z}^{\rho,-\rho,-\eta} f(z)=D_{z}^{-\rho} f(z)
$$

where $D_{z}^{-\rho} f(z)$ is the fractional integral of order $\rho$ of $f(z)$ which was introduced by Owa $([2],[3])$.

In order to prove our results for the fractional operator, we have to recall here the following lemma due to Srivastava, Saigo and Owa [6].

Lemma 2. If $\rho>0$ and $k>\delta-\eta-1$, then

$$
I_{0, z}^{\rho, \delta, \eta} z^{k}=\frac{\Gamma(k+1) \Gamma(k-\delta+\eta+1)}{\Gamma(k-\delta+1) \Gamma(k+\rho+\eta+1)} z^{k-\delta} .
$$


With the aid of Lemma 2, we have

Theorem 1. Let $\rho>0, \delta<2, \rho+\eta>-2, \delta-\eta<2$, and $\delta(\rho+\eta) \leq 3 \rho$.

If $f(z) \in K(n, \alpha, \beta)$, then

$$
\left|I_{0, z}^{\rho, \delta, \eta} f(z)\right| \geq \frac{\Gamma(2-\delta+\eta)|z|^{1-\delta}}{\Gamma(2-\delta) \Gamma(2+\rho+\eta)}\left\{1-\frac{(1+(1+2 n) \beta-2 n \alpha \beta)(2-\delta+\eta)_{n}(2)_{n}|z|^{n}}{(1+\beta)(n+1)^{2}(2-\delta)_{n}(2+\rho+\eta)_{n}}\right\}
$$

and

$$
\left|I_{0, z}^{\rho, \delta, \eta} f(z)\right| \leq \frac{\Gamma(2-\delta+\eta)|z|^{1-\delta}}{\Gamma(2-\delta) \Gamma(2+\rho+\eta)}\left\{1+\frac{(1+(1+2 n) \beta-2 n \alpha \beta)(2-\delta+\eta)_{n}(2)_{n}|z|^{n}}{(1+\beta)(n+1)^{2}(2-\delta)_{n}(2+\rho+\eta)_{n}}\right\}
$$

for $z \in U_{0}$, where

$$
U_{0}= \begin{cases}U & (\delta \leq 1) \\ U-\{0\} & (\delta>1)\end{cases}
$$

The result is sharp.

Proof. By using Lemma 2, we have

$$
I_{0, z}^{\rho, \delta, \eta} f(z)=\frac{\Gamma(2-\delta+\eta)}{\Gamma(2-\delta) \Gamma(2+\rho+\eta)} z^{1-\delta}-\sum_{k=n+1}^{\infty} \frac{\Gamma(k+1) \Gamma(k-\delta+\eta+1)}{\Gamma(k-\delta+1) \Gamma(k+\rho+\eta+1)} a_{k} z^{k-\delta}
$$

letting

$$
\begin{aligned}
H(z) & =\frac{\Gamma(2-\delta) \Gamma(2-\rho+\eta)}{\Gamma(2+\delta+\eta)} z^{\delta} I_{0, z}^{\rho, \delta, \eta} f(z) \\
& =z-\sum_{k=n+1}^{\infty} h(k) a_{k} z^{k},
\end{aligned}
$$

where

$$
h(k)=\frac{(2-\delta+\eta)_{k-1}(1)_{k}}{(2-\delta)_{k-1}(2+\rho+\eta)_{k-1}}, \quad(k \geq n+1) .
$$

We can see that $h(k)$ is non-increasing for integers $k \geq n+1$, and we have

$$
0<h(k) \leq h(n+1)=\frac{(2-\delta+\eta)_{n}(2)_{n}}{(2-\delta)_{n}(2+\rho+\eta)_{n}} .
$$

Since $f(z) \in K(n, \alpha, \beta)$, lemma 1 implies that $\sum_{k=n+1}^{\infty} a_{k} \leq \frac{(1+(1+2 n) \beta-2 n \alpha \beta)}{(1+\beta)(n+1)^{2}}$. Therefore, by using (2.10), we have

$$
\begin{aligned}
|H(z)| & =|z|-h(n+1)|z|^{n+1} \sum_{k=n+1}^{\infty} a_{k} \\
& \geq|z|-\frac{\{1+(1+2 n) \beta-2 n \alpha \beta\}(2)_{n}(2-\delta+\eta)_{n}}{(1+\beta)(n+1)^{2}(2-\delta)_{n}(2+\rho+\eta)_{n}}|z|^{n+1}
\end{aligned}
$$


and

$$
\begin{aligned}
|H(z)| & =|z|+h(n+1)|z|^{n+1} \sum_{k=n+1}^{\infty} a_{k} \\
& \geq|z|+\frac{\{1+(1+2 n) \beta-2 n \alpha \beta\}(2-\delta+\eta)_{n}(2)_{n}|z|^{n+1}}{(1+\beta)(n+1)^{2}(2-\delta)_{n}(2+\rho+\eta)_{n}}
\end{aligned}
$$

Sharpness follows if we take the function

$$
f(z)=z-\frac{\{1+(1+2 n) \beta-2 n \alpha \beta\}}{(1+\beta)(n+1)^{2}}|z|^{n+1} .
$$

Similarly by applying Corollary 1 to the function $f(z)$ belonging to the class $C^{*}(n, \alpha, \beta)$, we can derive

Theorem 2. Let $\rho>0, \delta<2, \rho+\eta>-2, \delta-\eta<2$ and $\delta(\rho+\eta) \leq 3 \rho$.

If $f(z) \in C^{*}(n, \alpha, \beta)$, then

$$
\begin{aligned}
\left|I_{0, z}^{\rho, \delta, \eta} f(z)\right| & \geq \frac{\Gamma(2-\delta+\eta)|z|^{1-\delta}}{\Gamma(2-\delta) \Gamma(2+\rho+\eta)} \\
& \left\{1-\frac{(1+(1+2 n) \beta-2 n \alpha \beta)(2-\delta+\eta)_{n}(2)_{n}|z|^{n}}{(1+\beta)(n+1)^{3}(2-\delta)_{n}(2+\rho+\eta)_{n}}\right\}
\end{aligned}
$$

and

$$
\begin{aligned}
\left|I_{0, z}^{\rho, \delta, \eta} f(z)\right| \leq & \frac{\Gamma(2-\delta+\eta)|z|^{1-\delta}}{\Gamma(2-\delta) \Gamma(2+\rho+\eta)} \\
& \times\left\{1+\frac{[1+(1+2 n) \beta-2 n \alpha \beta](2-\delta+\eta)_{n}(2)_{n}|z|^{n}}{(1+\beta)(n+1)^{3}(2-\delta)_{n}(2+\rho+\eta)_{n}}\right\}
\end{aligned}
$$

for $z \in U_{0}$ where $U_{0}$ is defined in Theorem 1

The equalities in (2.14) and (2.15) are attained by the function

$$
f(z)=z-\frac{(1+(1+2 n) \beta-2 n \alpha \beta)}{(1+\beta)(1+n)^{3}} z^{n} .
$$

\section{Convolution Product}

Let $f_{i}(z), \quad(i=1,2)$ be defined by

$$
f_{i}(z)=z-\sum_{k=n+1}^{\infty} a_{i, k} z^{k} \quad\left(a_{i, k} \leq 0\right) .
$$


We denote by $f_{1} * f_{2}(z)$ the convolution product of the functions $f_{1}(z)$ and $f_{2}(z)$ defined by

$$
f_{1} * f_{2}(z)=z-\sum_{k=n+1}^{\infty} a_{1, k} a_{2, k} z^{k}
$$

In order to show our results, we need the following

Theorem 3. A function $f(z) \in A(n)$ is in the class $K(n, \alpha, \beta)$ only if

$$
\sum_{k=n+1}^{\infty}\left\{(1+\beta)\left|k a_{k}-b_{k}\right|+2(1-\alpha) \beta b_{k}\right\} \leq 2 \beta(1-\alpha) .
$$

Proof. Let $|z|=r<1$. Noting

$$
\left|z f^{\prime}(z)-\varphi(z)\right|<\sum_{k=n+1}^{\infty}\left|k a_{k}-b_{k}\right| r
$$

and

$$
\left|z f^{\prime}(z)+(1-2 \alpha) \varphi(z)\right|>r\left\{2(1-\alpha)-\sum_{k=n+1}^{\infty}\left|k a_{k}-b_{k}\right|+2(1-\alpha) b_{k}\right\} .
$$

We see that

$$
\begin{aligned}
& \left|z f^{\prime}(z)-\varphi(z)\right|-\beta\left|z f^{\prime}(z)+(1-2 \alpha) \varphi(z)\right| \\
& \quad<r\left[\sum_{k=n+1}^{\infty}\left(\left|k a_{k}-b_{k}\right|(1+\beta)+2(1-\alpha) \beta b_{k}\right)-2(1-\alpha) \beta\right] .
\end{aligned}
$$

The right hand side of (3.4) is non-positive by (3.3) so that $f(z) \in K(n, \alpha, \beta)$.

Corollary 1. Let the function $f(z)$ defined by (1.1) be analytic in $U$. Then $f(z)$ is in $C^{*}(n, \alpha, \beta$,$) if and only if$

$$
\sum_{k=n+1}^{\infty}\left[(1+\beta) k\left|k a_{k}-b_{k}\right|+2(1-\alpha) \beta k b_{k}\right] \leq 2(1-\alpha) \beta .
$$

Theorem 4. Let the functions $f_{i}(z), \quad(i=1,2)$ be in the class $K(n, \alpha, \beta)$. Then $f_{1} * f_{2}$ belongs to the class $K(n, \gamma, \beta)$, where

$$
\gamma=\frac{[1+(1+2 n) \beta][(1+\beta) n+2(1+2 \alpha \beta)]-4 n \alpha^{2} \beta^{2}}{2 \beta(1+\beta)(1+n)^{2}} .
$$

The result if sharp. 
Proof. Employing the technique used earlier by Schild and Silverman [4], we need to find the largest $\gamma$ such that

$$
\sum_{k=n+1}^{\infty} \frac{(1+\beta)(1+n) k}{1+(1+2 n) \beta-2 n \gamma \beta} a_{1, k} a_{2, k} \leq 1
$$

It follows from (1.1) and the Cauchy-Schwarz inequality that

$$
\sum_{k=n+1}^{\infty} \frac{(1+\beta)(1+n) k}{1+(1+2 n) \beta-2 n \alpha \beta} \sqrt{a_{1, k} a_{2, k}} \leq 1
$$

Thus we need to find the largest $\gamma$ such that

$$
\frac{(1+\beta) k(n+1)}{1+(1+2 n) \beta-2 n \gamma \beta} a_{1, k} a_{2, k} \leq \frac{(1+\beta) k(n+1)}{1+(1+2 n) \beta-2 n \alpha \beta} \sqrt{a_{1, k} a_{2, k}}, \quad(k \geq n+1) .
$$

Or equaivlently, that

$$
\sqrt{a_{1, k} a_{2, k}} \leq \frac{1+(1+2 n) \beta-2 n \gamma \beta}{1+(1+2 n) \beta-2 n \alpha \beta}
$$

By virtue of (3.8) it is sufficient to find the largest $\gamma$ suth that

$$
\sqrt{a_{1, k} a_{2, k}} \leq \frac{1+(1+2 n) \beta-2 n \alpha \beta}{(1+\beta) k(n+1)}, \quad(k \geq n+1) .
$$

or, equivalently, that

$$
\gamma \leq \frac{k(1+n)(1+\beta)[1+(1+2 n) \beta]-[1+(1+2 n) \beta-2 n \alpha \beta]^{2}}{2 n \beta k(1+n)(1+\beta)}, \quad(k \geq n+1) .
$$

Since

$$
\phi(k)=\frac{k(1+n)(1+\beta)[1+(1+2 n) \beta]-[1+(1+2 n) \beta-2 n \alpha \beta]^{2}}{2 n \beta k(1+n)(1+\beta)}
$$

is an increasing function of $k$. Letting $k=n+1$ in (3.12) we obtain

$$
\gamma \leq \phi(n+1)=\frac{[1+(1+2 n) \beta][(1+\beta) n+2(1+2 \alpha \beta)]-4 n \alpha^{2} \beta^{2}}{2 \beta(1+\beta)(1+n)^{2}}
$$

which completes the proof of the theorem.

Finally, by taking the function given by

$$
f_{i}(z)=z-\frac{1+(1+2 n) \beta-2 n \alpha \beta}{(1+n)^{2}(1+\beta)} z^{n+1}, \quad(i=1,2),
$$

we can see the result is sharp. 
Similarly, we have

Theorem 5. Let the function $f_{i}(z), \quad(i=1,2)$ defined by (3.1) be in the class $C^{*}(n, \alpha, \beta)$. Then $f_{1} * f_{2}(z)$ belongs to the class $C^{*}(n, \gamma, \beta)$, where

$$
\gamma=\frac{(1+\beta)(1+n)^{3}[1+(1+2 n) \beta]-[1+(1+2 n) \beta-2 n \alpha \beta]^{2}}{2 n \beta(1+\beta)(1+n)^{3}} .
$$

The result is sharp for the functions given by

$$
f_{i}(z)=z-\frac{1+(1+2 n) \beta-2 n \alpha \beta}{(n+1)^{3}(1+\beta)} z^{n+1}, \quad(i=1,2) .
$$

Theorem 6. Let the functions $f_{i}(z), \quad(i=1,2, \ldots, m)$ defined by $(3.1)$ be in the class $K(n, \alpha, \beta)$. Then the function

$$
h(z)=z-\sum_{k=n+1}^{\infty}\left(a_{1, k}^{2}+a_{2, k}^{2}+\cdots+a_{m, k}^{2}\right) z^{k}
$$

belongs to the class $K(n, \gamma, \beta)$, where

$$
\gamma=\frac{[1+(1+2 n) \beta]\left\{(1+\beta)(1+n)^{2}-m[1+\beta(1+2 n(1-\alpha))]\right\}-4 m n^{2} \alpha^{2} \beta^{2}}{2 n \beta(1+\beta)(1+n)^{2}}
$$

The result is sharp for the functions $f_{i}(z), \quad(i=1, \ldots, m)$ defined by (3.15).

Proof. By virtue of Lemma 1, we obtain

$$
\sum_{k=n+1}^{\infty} \frac{(1+\beta)^{2} k^{2}(1+n)^{2}}{(1+(1+2 n) \beta-2 n \alpha \beta)^{2}}\left(a_{1, k}^{2}+a_{2, k}^{2}+\cdots+a_{m, k}^{2}\right) \leq m .
$$

Therefore we need to find the largest $\gamma$ such that

$$
\frac{(1+\beta)(1+n) k}{1+(1+2 n) \beta-2 n \gamma \beta} \leq \frac{1}{m} \frac{(1+\beta)^{2} k^{2}(1+n)^{2}}{(1+(1+2 n) \beta-2 n \alpha \beta)^{2}}, \quad(k \geq n+1)
$$

that is,

$$
\gamma \leq \frac{(1+\beta) k(1+n)[1+(1+2 n) \beta]-m[1+(1+2 n) \beta-2 n \alpha \beta]^{2}}{2 n \beta(1+\beta) k(1+n)}, \quad(k \geq n+1) .
$$

Just as in the proof of Theorem 4, we conclude that

$$
\gamma \leq \varphi(n+1)=\frac{[1+(1+2 n) \beta]\left\{(1+\beta)(1+n)^{2}-m[1+\beta(1+2 n(1-\alpha))]\right\}-4 m n^{2} \alpha^{2} \beta^{2}}{2 n \beta(1+\beta)(1+n)^{2}}
$$

and Theroem 6 follows at once. 


\section{Acknowledgement}

The author is thankful to the referee for his helpful criticism and comments.

\section{References}

[1] V. P. Gupta, Convex class of starlike functions, Yokohama Math. J. 32(1984), 55-59.

[2] S. Owa, On the distortion theorems, Kyungpook Math. J. 18(1978), 53-59.

[3] S. Owa, Some applications of the fractional calculus, Research Notes in Math. 187, Pitman, Boston, London and Melbourne, (1985), 164-175.

[4] A. Schild and H. Silverman, Convolutions of univalent functions with negative coefficients, Ann. Univ. Mariae Currie-Sklodowska Sect. A 29(1975), 99-107.

[5] H. M. Srivastava, M. Saigo and S. Owa, A calss of distortion theorems involving certain operators of fractional calculus, J. Math. Anal. Appl. 131(1988), 412-420.

Department of Mathematics, Faculty of Science, Girl College Dammam, Saudi Arabia. 